\title{
The Diagnostic Dilemma of Isolated Cardiac Sarcoidosis
}

\author{
Yoshikazu Yazaki
}

Key words: cardiac sarcoidosis, endomyocardial biopsy, diagnosis

(Intern Med 52: 1-2, 2013)

(DOI: 10.2169/internalmedicine.52.9008)

Sarcoidosis is a granulomatous disease of unknown etiology involving various organs including the heart. Cardiac involvement is one of the important prognostic factors in patients with sarcoidosis (1). There are two clinical scenarios in the initial presentation of cardiac sarcoidosis (CS). First, it is not very difficult to diagnose cardiac involvement in patients with known extra-cardiac sarcoidosis, because careful follow-up with 12-lead electrocardiography supported by echocardiography enables early detection of CS. The second scenario includes patients admitted for cardiac signs and symptoms as the first manifestation of sarcoidosis. If there is no clinical evidence of sarcoidosis in other organs, it is not easy to confirm the diagnosis in such patients so-called "isolated CS" (2). Although histopathological confirmation is needed (3), the sensitivity of endomyocardial biopsies is less than $20 \%$ due to the random distribution of cardiac involvement. Fortunately, the case reported by Sugizaki et al. (4) involved histological confirmation of CS on an endomyocardial biopsy, and that patient was successfully treated with corticosteroids. However, such cases are rare, and it is quite difficult to establish a clinical diagnosis of isolated CS.

When inflammatory infiltrates with giant cells are confirmed on endomyocardial biopsies, obtaining histological confirmation and differentiation of CS from giant cell myocarditis (GCM) should be required because both disease entities present with similar cardiac manifestations. In general, granuloma formation by epithelioid cells is necessary for the development of CS. Okura et al. (5) carefully compared the histopathology of CS and GCM and demonstrated that fibrosis and granulomas are prominent in CS, whereas necrosis and eosinophils are prominent in GCM. A photomicrograph reported by Sugizaki et al. (4) seems to demonstrate granulomatous inflammation, although the authors did not explain the characterization of the inflammatory infiltrates.

Recently, new imaging modalities such as cardiac magnetic resonance imaging (CMR) and ${ }^{18} \mathrm{~F}$-fluorodeoxy glucose $\left({ }^{18} \mathrm{~F}\right.$-FDG) positron emission tomography (PET) have been introduced in the diagnosis and management of patients with CS $(6,7)$. Using CMR, patients with established extracardiac sarcoidosis can be increasingly screened for asymptomatic cardiac involvement (6). Late gadolinium enhanced (LGE) images highlight areas of inflammation and fibrosis as regions of increased signal intensity. The patterns and locations of LGE are variable in patients with CS. LGE is preferentially observed in the mid to epicardial layer in CS patients and in the endocardial layer in patients with myocardial infarction, although the case reported by Sugizaki et al. involved LGE in the endocardial layer. LGE can be observed in any territory and as any pattern in patients with CS. CMR has the potential to be an important tool for the early diagnosis of CS. ${ }^{18}$ F-FDG accumulation correlates with the histological activity of sarcoidosis. In a direct comparison of gallium-67 scintigraphy (7), PET with ${ }^{18} \mathrm{~F}-\mathrm{FDG}$ is significantly more sensitive than gallium-67. The improved sensitivity is offset by a loss in specificity. Uptake of ${ }^{18} \mathrm{~F}$ FDG in the myocardium not only indicates inflammation, but also normal glucose metabolism. Usually, ${ }^{8}$ F-FDG uptake should be evaluated concomitant with myocardial perfusion images. It should be noted that the specificity of PET with ${ }^{18}$ F-FDG in detecting cardiac sarcoidosis remains unclear. ${ }^{18}$ F-FDG PET has the potential to be an important tool for monitoring patients with cardiac sarcoidosis.

The Japanese Ministry of Health and Welfare first proposed guidelines for the diagnosis of CS in 1992, which were revised in 2006. Increasing recognition of clinically isolated CS and recent advances in imaging modalities will lead to the need for further revisions of the diagnostic criteria.

The author states that he has no Conflict of Interest (COI).

\section{References}

1. Yazaki $Y$, Isobe $M$, Hiroe $M$, et al. Prognostic determinants of long-term survival in Japanese patients with cardiac sarcoidosis 
treated with corticosteroids. Am J Cardiol 88: 1006-1010, 2001.

2. White J, Sutton T, Kerr A. Isolated primary cardiac sarcoidosis: MRI diagnosis and monitoring of treatment response with cardiac enzyme. Circ Heart Fail 3: e28-e29, 2010.

3. Kandolin R, Lehtonen J, Graner M, et al. Diagnosing isolated cardiac sarcoidosis. J Intern Med 270: 461-468, 2011.

4. Sugizaki Y, Tanaka H, Imanishi J, et al. Isolated primary cardiac sarcoidosis presenting as acute heart failure. Internal Med 52: 7174, 2013.
5. Okura Y, Dec GW, Hare JM, et al. A clinical and histopathological comparison of cardiac sarcoidosis and idiopathic giant cell myocarditis. J Am Coll Cardiol 41: 322-329, 2003.

6. Patel MR, Cawley PJ, Heitner JF, et al. Detection of myocardial damage in patients with cardiac sarcoidosis. Circulation 120: 1969-1977, 2009.

7. Okumura W, Iwasaki T, Toyama $\mathrm{T}$, et al. Usefulness of fasting ${ }^{18} \mathrm{~F}-$ FDG PET in identification of cardiac sarcoidosis. J Nucl Med 45: 1989-1998, 2004.

(C) 2013 The Japanese Society of Internal Medicine http://www.naika.or.jp/imonline/index.html 